\title{
Cognitive information processing
}

\author{
Chi Tran
}

Received: 12 December 2013 / Accepted: 8 March 2014 / Published online: 30 April 2014

(C) The Author(s) 2014. This article is published with open access at Springerlink.com

\begin{abstract}
Today, under the "true or false" philosophy, we are confined within the 'syntactic' restrictions, in connection with the adoption of the counter-concepts/binary concepts presented by common words and Boolean numbers concerned with logical reasoning based on binary logic. In contrast, truth, according to Brouwer and Heyting (intuitive logic), comes from the future. It, according to Łukasiewicz (multi-valued logic), is neither true nor false. It should be a third option that transcends both, i.e., transcends the 'syntactic' restrictions to reach the 'semantic' and 'pragmatic' descriptions of available data. Truth, in this case, will be formulated in the new form of the meta-concept: true-false which is neither true nor false, likewise the 'affirmation and denial' idea of the Indian logic. It becomes a cognitive measure used to describe the human cognitive processing. It turned out that we need not only a new term that transcends limits of words and Boolean numbers to describe meta-concepts, but also a new approach based on new philosophy, logic and mathematics to process cognitive information using both physical and mental data. It refers, in this article, as denotation computing for determining the relative density of sand using CPT data. Instead of the counter-concept, "loose or consolidation or high consolidation" we use the meta-concept, "loose-consolidation-high consolidation" to represent the objective-subjective data. Their processing is developed for certain types of data structures designed for representing information content including cognitive measures depending on perception of engineers to exceed the syntax limits of binary concepts towards their semantic and pragmatic aspects. It is based on the integration
\end{abstract}

C. Tran $(\varangle)$

Faculty of Technical Sciences, WM University, Olsztyn, Poland

e-mail: tran.chi@uwm.edu.pl of modern philosophy, epistemology, logic, and denotational mathematics.

Keywords Geotechnical engineering - Counter-concept . Meta-concept - Cognitive measure · Cognitive information processing $\cdot$ Denotational mathematics

\section{Introduction}

To identify what we consider the so-called objective reality through available data derived from the physical devices, we use traditionally logical reasoning within the 'syntactic' restrictions, which is based on the binary philosophy and two-valued logic. It we call the information processing in the 'syntactic' level-external information processing using the counter-concept/binary concept: 'true or false'the first stage of our relationship with the real world. In contrast, when we experience what we feel a reality, which may extend beyond the boundary of syntax restrictions of language. For example, we have some objects/subjects which we do not know for sure of its existence or non-existence. However, we feel that it is neither existence nor non-existence, but a third option that transcends both. To describe what we have (feel) in our head, we may introduce a new concept originated from intuition and experiences depending on our perception derived from the so-called true-false philosophy according to monism rather than dualism. We cannot use common words with syntax restriction to describe this idea. We may use a new concept presented by existencenonexistence, which represents both existence and nonexistence. It we call a meta-concept, which is derived from our intuition, commonsense and experiences. Hidden information containing in this existence-nonexistence concept, we call cognitive information, which represents the semantic and 
pragmatic aspects of observed object/subject. Thus, we have also a relationship with the real world - the second stage, which is strictly related to cognitive processing using cognitive measures. Then, we need a new technique that studies information as attributes of the real world, which shall be generally abstracted, quantitatively represented and mentally processed.

We propose in this paper, an internal information processing approach based on the integration of modern philosophy, epistemology, logic and denotational mathematics for certain types of data structures designed for representing information content including cognitive measures derived from intuition, commonsense and perception of engineers to exceed the syntax limits of binary concepts towards their semantic and pragmatic aspects. It refers as denotation computing using both physical and mental data to determine the relative density, $I_{D}$, of sands with cognitive measures determined by 'true-false' values. Instead of the counter-concept "loose or medium or high consolidation" according to dualism, we use rather the meta-concept, "loose-medium-high" consolidation, determined by both available data and mental data according to monism.

\section{Philosophy of language}

Linguists, in the nineteenth century, began to recognize the diversity of human language, and to question about the relationship between language and logic. Different concepts have been introduced, for example, syntactic, semantics, pragmatics, sense and denotation which are presented briefly as below.

Syntactic or syntax, is concerned with the way sentences are constructed from smaller parts, such as words and phrases. Two steps can be distinguished in the study of syntactic. The first step is to identify different types of units in the stream of speech and writing. The second step is to analyze how these units build up larger patterns, and in particular to find general rules that govern the construction of sentences. Syntactic is a branch of semiotics that deals with the formal relations between signs or expressions in abstraction from their signification and their interpreters.

Semantics is the subfield that is devoted to the study of meaning, as inherent at the levels of words, phrases, sentences, and larger units of discourse. A key concern is how meaning attaches to larger chunks of text, possibly as a result of the composition from smaller units of meaning. Traditionally, semantics has included the study of sense and denotation reference, truth conditions, argument structure and discourse analysis. The term semantics is applied to certain types of data structures, specifically designed and used for representing information content. Systems of categories are not

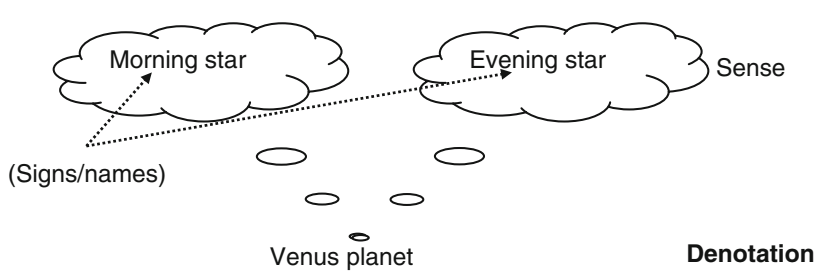

Fig. 1 Sense and denotation according to Frege's view

objectively "out there" in the world, but are rooted in people's experiences. Their meaning is not an objective truth, but a subjective construct, learned from experiences.

Pragmatics is the study of the relationships between the symbols of a language, their meaning, and the users of the language. It is a subfield of linguistics which studies the ways in which context contributes to meaning. It studies how the transmission of meaning depends not only on the linguistic knowledge of the speaker and listener, but also on the context of the utterance, knowledge about the status of those involved, the inferred intent of the speaker, and so on. In this respect, pragmatics explains how language users are able to overcome apparent ambiguity, since the meaning relies on the manner, place, time etc., of an utterance. Pragmatic awareness is regarded as one of the most challenging aspects of language learning, and comes only through experiences.

Sense and denotation Frege (1848-1925) [1,2] developed a theory of sense and denotation into a thoroughgoing philosophy of language. He gave a new and revised account of his logical calculus, and had evolved his now well-known theory of sense and denotation. By the sense of a statement or proposition, Frege understood its content, i.e., 'the meaning of words' in the syntactic level. While by denotation of a statement or proposition he understood the meaning, in the semantic and pragmatic levels, that transcends the language description. According to Frege, we can really learn that the statement "the morning star is identical to the morning star" is true by simply linguistic inspection, but, while, in fact both the statements "the morning star" and "the evening star" refer to is the planet namely Venus, we cannot learn the truth that "the morning star is identical to the evening star" simply by linguistic inspection. It is shown in the Fig. 1.

\section{Counter-concepts/binary-concept}

The counter-concepts such as mind or brain, being or nonbeing, objectivity orsubjectivity and others are originated from dualism, which was originally created to denote coeternal binary opposition, a meaning with syntactic restrictions that are preserved in metaphysical and philosophical duality discourse. For example, 'mind or brain' dualism claims that neither the mind nor brain can be reduced to each other in any way. This alternative idea, as well as 


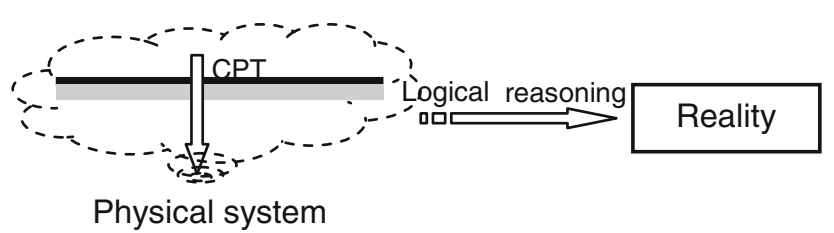

Fig. 2 Objective reality derived from available data

counter-concepts such as being or nonbeing introduced later creates the so-called binary philosophy-Aristotle (344-322 BC, see Barnes, Jonathan). Here, "Being" and its opposition, "Nonbeing", are expressed in the law of the excluded middle, which states the necessity that either an assertion or its negation must be true or false.

Counter-concepts originating from 'being or nonbeing' philosophy are derived from observation, physical testing and logical reasoning based on two-valued logic, in which we have the so-called material implication presented logically in the form:

$\forall_{p, q \in\{0,1\}} p \rightarrow q=0$ or 1 (certainly)

Meaning of these concepts can be determined by a common language in its syntactic aspects, without its semantic and pragmatic respects depending upon the individualized conscious or actual spiritual state of the observer. Consider, for example, behaviors of explored equipment such as the Cone Penetration Test, CPT, in the complex environments of soil. Available knowledge about soil properties can be obtained from observation of the behavior of the physical "CPT-soil" system and logical reasoning including traditional/deductive mathematics. It is seen as exploration strictly in the framework of the 'syntactic' restrictions, depending in no way upon the individual spiritual state of the controller, which is shown in the Fig. 2.

Study of the "CPT-soil" system must, in this case, resort to statistical descriptions and what has been determined is seen as 'objectivity'. Then, the laws of statistics enable us to understand the behavior of a multitude of disorganized complexities.

\section{Meta-concept}

Contrary to dualism, monism does not accept any fundamental divisions between physical and mental. It is a doctrine saying that ultimate it reality is entirely of 'one' substance. Monism [3] leads to other concepts named metaconcepts, which were originally created to denote a meaning beyond syntactic restrictions that are preserved in metaphysical, philosophical duality discourse. They are presented as follows.

'Affirmation-Denial' Concept: It was found in Indian logic (in the Rigveda 1000 B.C.) in which, we have the four-

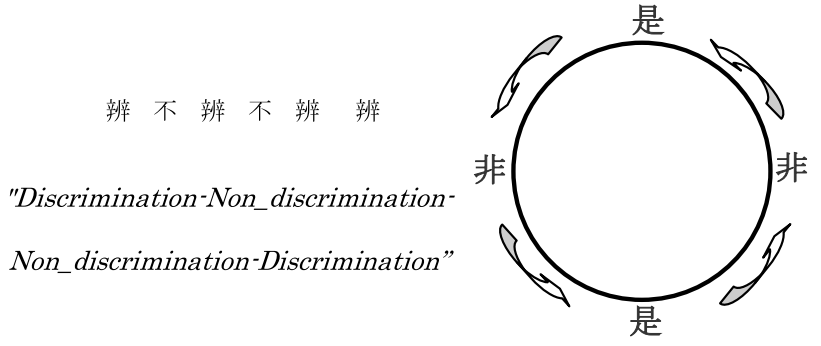

Fig. 3 Zhuang Zhou's “One” philosophy

cornered argumentation including: affirmation $\boldsymbol{X}$, denial of $X, \neg X$, both affirmation and denial, $X \wedge \neg X$, and neither of them $\neg(X \wedge \neg X)$. Here, the joint affirmation and denial $X \wedge \neg X$ represents a third option that transcends both affirmation and denial. It can be represented by a new metaconcept: 'affirmation-denial' in the form:

'affirmation-denial' is neither affirmation nor denial

It is impossible in two-valued logic, i.e., we cannot use it for logical reasoning based on binary logic. The efforts of Indian logic were concentrated on explaining the unary underlying order of Hindu philosophy. They want to come to adopt neutral monism, the view that the possible reality, which we could recognize by feeling originated from intuition, commonsense, is of one kind including both affirmation and its denial.

'Discrimination-Nondiscrimination' Concept: Fundamental undivided nature of reality is found in Zhuang Zhou's 'One' philosophy, (莊 周-Zhuang Zhou 369-286, in China [4]). It is shown in the Fig. 3.

The author claimed that the concept of true and the opposition of it, false, is seen as oneness and according to his dynamic thinking: "true becomes false and false becomes true ... we cannot know where is the starting point, where is the end ... as a circle, in which, we cannot distinguish big from small, good from evil, discrimination from non-discrimination and true from false". The original idea presented in brief in the form: "DiscriminationNondiscrimination", is seen as the Zhuang Zhou's 'One' philosophy:

'Discrimination-Nondiscrimination' is neither Discrimination nor Nondiscrimination,

which, according to Suzuki [5] (鈴木 大拙 貞太郎 Suzuki Daisetz Teitaro 1962), is the most deep philosophical paradox. In fact, the author wanted to turn away from logical reasoning to recognize what originated directly from the observer's perceptions.

'Being-Nonbeing' Concept: The meta-concept: "sac-sackhong-khong" i.e., being-being-nonbeing-nonbeing or briefly, being-nonbeing is found in the Vietnamese "one" philosophy. That is: 
'being-nonbeing' is neither being nor nonbeing

but a third option that transcends both. In the framework of Indian logic, using box connective, $\square$ (affirmation), and diamond connective, $\boldsymbol{\nabla}$ (both $\square$ and $\neg \boldsymbol{\square}$ ), for the fourcornered argumentation: $\boldsymbol{\square}, \boldsymbol{\nabla}, \neg \mathbf{\square}$ and $\neg \boldsymbol{\nabla}$, we can represent 'Being' by four symbols, $(\boldsymbol{\square}, \boldsymbol{\nabla}, \neg \boldsymbol{\square} B, \neg \diamond B)$, where we have certainty: $\square B, \neg \square B$ (negation), uncertainty: $\diamond B$ and $\neg \diamond B$ (neither $\square B$ nor $\neg \square B$ ); similarly, 'Nonbeing' by $(\square N, \diamond N, \neg \square N, \neg \nabla N)$. Then, being-nonbeing reasoning based on Indian logic (four-valued logic) leads to 16 possibilities, which are presented in the form:

Is this "being-nonbeing"?

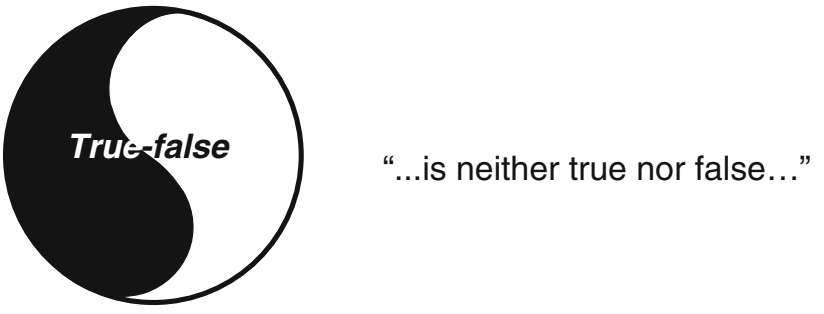

Fig. 4 Łukasiewicz's 'true-false' philosophy

ultimately the structures of thought and reality, subject and object, are identical. According to Hegel, people do not recognize that, in truth, the identity is different, that is to say, they are "one" — the identity-different.

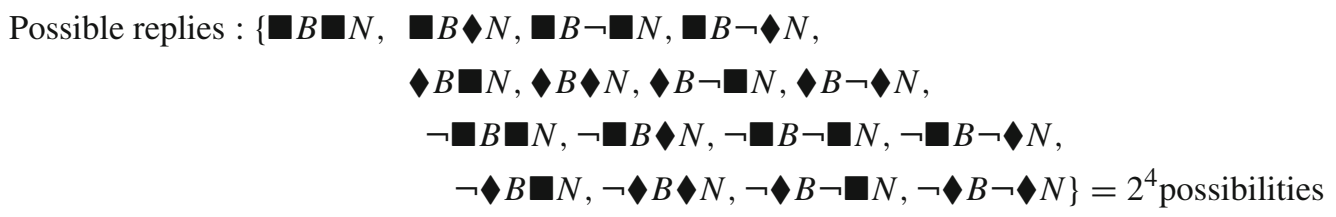

This is a consequence of both the observations that come from the eyes of the observer and his/her perceptions rooted from intuition, commonsense and experiences without logical reasoning, i.e., beyond the syntactic boundaries by a cognitive information processing.

While the counter-concepts 'being or nonbeing' are produced by the intercourse of the body senses with objects, the observer can quickly recognize being or non-being by his/her eyes. Consequently, four possibilities obtained from 'being ornonbeing' reasoning based on two-valued logic are presented in the form:

Is this "being or nonbeing"?

Possible replies: $\{B N, B \neg N, \neg B N, \neg B \neg N\}=2^{2}$ possibilities

Thus, with the meta-concept: being-nonbeing, we can recognize many more possibilities, which we cannot obtain by logical reasoning based on two-valued logic. Many possibilities individually exist in the observer's spirit through actual information and his/her perceptions using 'to look inside' information processing - 'true and false' reasoning rather than 'true or false' reasoning.

It is the conscious development [6], which defines the reality of semantic and pragmatic aspects using mental power of consciousness of the observer rather than a reality in terms of syntax derived from "being or non-being" reasoning.

'Subject-Object' concept: According to Hegel, the most important achievement of German idealism, starting with Kant and culminating in his own philosophy, was "the demonstration that reality is shaped through and through by mind and, when properly understood, is mind". Thus,
'True-false' Concept: The meta-concept true-false presented in Łukasiewicz's philosophy (1879-1956) as in [7]: "The truth about the future events is neither true nor false", i.e., it comes from intuition and commonsense or from the human cognitive processing depending on perceptions rather than 'true or false' reasoning in the syntactic framework. It is a cognizable value - 'true-false' measure, $\tau$, and the 'truth' of judgments or the cognitive measures, which is graphically presented in the form Fig. 4.

According to Łukasiewicz, for a cognitive measure, $\tau, \tau=$ $\mathrm{p}, \mathrm{q} \in[0,1]$, instead of the material implication, we have a strict implication presented as:

$\forall_{p, q \in[0,1]} p \rightarrow q=\min (1,1-p+q)$ (uncertainly)

'Membership-nonmembership' concept: It is neither membership nor nonmembership [8], which is developed and leads to Zadeh's fuzzy logic.

Information Content of Meta-concepts: According to Frege, the denotation of statement, p, must be identified by truth values or an infinite number of thoughts (cognitive processing). That is:

$$
\mathrm{p} \Rightarrow \mathrm{p} \mid \text { Denotation }=\mathrm{p} \mid \text { thoughts }
$$

Thought that comes to mind quickly without much personal reflection is a consequence derived from intuition, commonsense through perceptions, which is often roughly translated as 'feeling or to look inside'. It really describes a 'subjective evaluation'/'cognitive evaluation' in the semantic and pragmatic aspects-information content that exists in different ideas: 'Discrimination-Nondiscrimination' accord- 
ing to Zhuang Zhou' philosophy, 'being-nonbeing' according to Vietnamese philosophy, 'identity-different' according to Hegel's philosophy, 'membership-nonmembership' according to Zadeh's philosophy, 'true-false' according to Lukasiewicz's philosophy and 'truth comes from the future' according to intuitive logic of Brouwer and Heyting $[9,10]$. By this way, we can represent the meta-concept affirmationdenial as follows

affirmation-denial $\mid$ thoughts = affirmation-denial $\mid \operatorname{cog}$ nitive measures

The True-false measure, $\tau$, referred as cognitive measure enables us to represent the semantic and pragmatic aspects of knowledge through the human pragmatic competences. Data with its cognitive measure, we call intelligent data and the knowledge derived from intelligent data we call the metaknowledge. These can be estimated by how much it resonates with our being through an internal processing (feeling) of the human mind. We have, according to multi-valued logic, truer if fewer false and vice versa; that is:

$$
\begin{aligned}
\text { True }- \text { false }= & \text { True }- \text { false } \mid \tau(\text { true }) \\
& \wedge \text { True }- \text { false } \mid \tau(\text { false })
\end{aligned}
$$

In the same way, we have:

$$
\begin{aligned}
& \text { Affirmation-denial }=\text { Affirmation-denial } \mid \tau \quad(\text { affirma- } \\
& \text { tion }) \wedge \text { Affirmation-denial } \mid \tau(\text { denial }) \\
& \text { Being-Nonbeing = Being-Nonbeing } \mid \tau(\text { being }) \wedge \text { Being- } \\
& \text { Nonbeing } \mid \tau \text { (Nonbeing) }
\end{aligned}
$$

These data structures are actually intended to represent the information content through cognitive measures, $\tau$, determined under the epistemological aspect of engineers to expand the syntactic boundaries of available data into the semantic and pragmatic aspects. It is the result of quantity expressed by numbers through cognitive measures, quality expressed by words through meta-concepts and the human thought through cognitive information processing. Their integration held in relation to Frege's concept of sense and denotation of sentences and Lukasiewicz's multi-valued logic.

Let us return to geotechnical problems, it is seen as an exploration in the framework that transcends the 'syntactic' restrictions, depending on the individual spiritual state of the observer and scientist, which is shown in the Fig. 5.

\section{Cognitive information processing using denotational mathematics}

Note, that the symbol $\tau$, in the framework of the fuzzy philosophy, is called a truth function that expresses the truth according to the degree of truth. In contrast, in the frame-

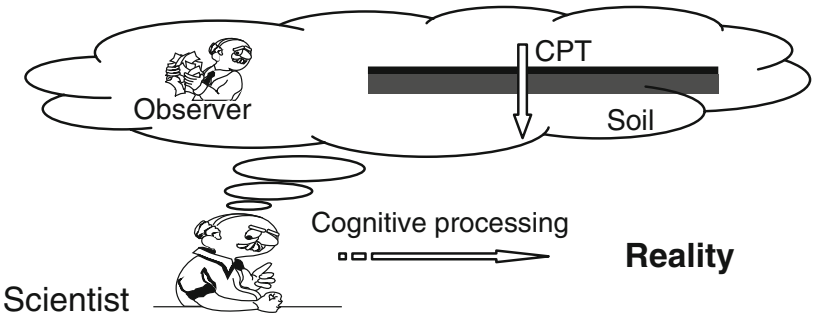

Fig. 5 Meta-knowledge that expresses objective-subjective reality

work of "being-nonbeing" of philosophy, it is called "truefalse" function referred as the cognitive measures. Their processing - cognitive measure processing can be mathematically represented as follows.

Definition 1 Borel $\sigma$-algebra: let $\mathrm{X}$ be a non-empty set, $\mathrm{X} \neq \varnothing$; the family $\mathbb{A}$ of subsets of the set $\mathrm{X}$ is called $\sigma$-algebra of subsets of $\mathrm{X}$, if:

$$
\begin{gathered}
(\mathrm{X} \in \mathbb{A}) \wedge(\mathrm{A} \in \mathbb{A} \rightarrow(\mathrm{X}-\mathrm{A}) \in \mathbb{A}) \\
\wedge\left(\mathrm{A}_{\mathrm{n}} \in \mathbb{A} \rightarrow \bigcup_{n=1}^{\infty} A_{n} \in \mathbb{A}\right)
\end{gathered}
$$

The $\sigma$-algebra, which is generated by all open intervals in $\Re^{\mathrm{n}}$, is called Borel $\sigma$-algebra and denoted as $ß$. The elements of family $B$ are called Borel sets.

Definition 2 Measure: a function $\tau: \mathbb{A} \Rightarrow \bar{\Re}_{+}, \bar{\Re}_{+}=\Re \cup$ $\{+\infty\}$ is called a measure, if:

(a) $\forall(A \in \mathbb{A}):\{(\tau(\mathrm{A}) \geq 0) \wedge(\tau(\varnothing)=0)\}$

(b) $\forall_{A_{i} \cap A_{j}=\varnothing ; i \neq j}:\left\{\tau\left(\bigcup_{i=1}^{\infty} A_{i}\right)=\sum_{i=1}^{\infty} \tau\left(A_{i}\right)\right\}$

Definition 3 Measure of a set: The function $\tau(A), A \in \mathbb{A}$ is called a measure of set $A$; if $\tau(A)=0$ then $A$ is called a measurable-zero set, $\tau: \mathbb{A} \rightarrow[0, \infty]$ is called a set function determined on $\mathbb{A}$.

Definition 4 Measurable set: Assume, family $\Im$ composed of elements in the form of: $A=B \cup C, B \in \beta, \tau(\mathrm{C})=0$; $\beta$ is a Borel set; each element of set $\mathfrak{I}$ is called a measurable set (measurable in the sense defined by Lebesgue [11]), if:

$\forall_{\mathrm{A} \in \mathfrak{I}}: \tau(\mathrm{A})=\tau(\mathrm{B} \cup \mathrm{C})=\tau(\mathrm{B})$

Definition 5 Measurable function: mathematically, let X be a non-empty set, let $\mathrm{S}$ be a $\sigma$-field of subsets of $\mathrm{X}$, let $f$ be a partial function from $\mathrm{X}$ to $, \bar{\Re}, \bar{\Re}=\Re \cup\{-\infty\} \cup\{+\infty\}$ and let $A$ be an element of S. We say that $f$ is measurable on $A$ iff: for every real number $r$ holds, as in [12].

$\mathrm{A} \cap \mathrm{LE}-\operatorname{dom}(\mathrm{f}, \bar{\Re}(\mathrm{r}))$ is measurable on $\mathrm{S}$ 
where, the functor LE-dom $(f$, a) yields a subset of $\mathrm{X}$ and is defined by:

$x \in \mathrm{LE}-\operatorname{dom}(f$, a)iff: $x \in \operatorname{dom}(f) \wedge \exists \mathrm{y}\{y=f(x) \wedge y<\mathrm{a}\}$,

where $y$ denotes an extended real number. In other way, let $F$ be a class of all finite non-negative measurable functions defined on a measurable space, $(X, \mathbb{A})$. Moreover, let the set $\mathrm{F}_{\alpha}$ be called an $\alpha$-cut of $f, f \in \mathrm{F}$. Function $f: \mathrm{X} \Rightarrow \bar{\Re}$ is called a measurable function iff: $\beta$ is a Borel set and:

$$
\begin{aligned}
& \forall_{B \in \beta}:\left\{f^{-1}(B)=[x: f(x) \in B] \text { belonging to } \sigma\right. \\
& \quad \text {-algebra on } X\} \text { albo } \\
& \left\{\forall_{\alpha \in \Re}: F_{\alpha}=[x: f(x)>\alpha]\right\}
\end{aligned}
$$

Definition 6 Quantitative-qualitative evaluation: Let X be a non-empty set; $\mathrm{A}$ be an $\sigma$-algebra on $\mathrm{X} ; \tau: \mathbb{A} \Rightarrow[0,1]$ be a non-negative, real-valued set function defined on $A . \tau$ is called a cognitive measure on $(X, \mathbb{A})$ iff:

a. $\tau$ is derived from available data with the respect of semantics and pragmatics.

b. $\tau(\varnothing)=0$ when $\varnothing \in \mathbb{A}$.

c. $\{(\mathrm{E} \in \mathbb{A}, \mathrm{F} \in \mathbb{A}) \wedge(\mathrm{E} \subset \mathrm{F})\}$ implies $\tau(\mathrm{E}) \leq \tau(\mathrm{F})$.

d. $\left[\left(\left\{E_{n}\right\} \subset \mathbb{A} ; \cup\left\{E_{n}\right\} \in \mathbb{A}\right) \wedge\left(\cap\left\{E_{n}\right\} \in \mathbb{A}\right)\right]$ then

$$
\left[\tau\left(\cup\left\{E_{n}\right\}\right)=\max \left\{\tau\left(E_{n}\right)\right\}\right] \wedge\left[\tau\left(\cap\left\{E_{n}\right\}\right)=\min \left\{\tau\left(E_{n}\right)\right\}\right]
$$

This number is the quantitative-qualitative evaluation (a cognitive measure) corresponding to both quantity and quality factors in $\mathrm{X}$. Hence, set $\varnothing$ has a minimum confidence: 0 , i.e., $\tau(\varnothing)=0$; total space $\mathrm{X}$ has a maximum confidence: 1, i.e., $\tau(X)=1$ (boundary conditions); therefore, for every set of factors $E$ i $F$, so that $\mathrm{E} \subset \mathrm{F}$, the confidence of $\mathrm{E}$ cannot be greater than the confidence of $\mathrm{F}$. This means that this measure fulfills the conditions: $\mathrm{E} \in \mathbb{A}, \mathrm{F} \in \mathbb{A}$ and $\mathrm{E} \subset \mathrm{F}$ implies $\tau(\mathrm{E}) \leq \tau(\mathrm{F})$ (monotonicity conditions). Measure $\tau$ corresponding to above conditions is called a true-false measure-fuzzy measure in the measurable space $(X, \mathbb{A})$. Compared to a probabilistic measure, the additive condition $(\tau(\mathrm{E} \cup \mathrm{F})=\tau(\mathrm{E})+\tau(\mathrm{F})$ for $\mathrm{E} \cap \mathrm{F}=\varnothing)$ has been replaced by condition (c); therefore, we call it $(\tau)$ a non-additive measure. $\tau$ is called a lower or upper semi-continuous true-false measure on $(X, \mathbb{A})$ iff it satisfies the above conditions [a, $\mathrm{b}$ and $\mathrm{c}$ (continuity from below)] or [a, b and d (continuity from above)], respectively. Both of them are simply called semi-continuous true-false measures. Furthermore, we say that true-false measure or semi-continuous true-false measure, $\tau$, is regular iff $X \in \mathbb{A}$ and $\tau(X)=1$. A set function $\tau$ is called true-false -additive measure iff $g: \mathbb{A} \rightarrow[0,1]$ on space $(X, \mathbb{A})$ such that $\tau\left(\cup_{t \in T} E_{t}\right)=\sup _{t \in T} \tau\left(E_{t}\right)$ for any subclass $\left\{E_{t} \mid t \in T\right\}$ of $\mathbb{A}$ whose union is in $\mathbb{A}$, where $T$ is an arbitrary index set. For any $E, F \subseteq X$, a true-false measure is:

1. additive: $\forall E \cap F=\varnothing\{\tau(E \cup F)=\tau(E)+\tau(F)\}$;

2. supermodular: $\tau(E \cup F)+\tau(E \cap F) \geq \tau(E)+\tau(F)$;

3. submodular: $\tau(E \cup F)+\tau(E \cap F) \leq \tau(E)+\tau(F)$;

4. superadditive: $\forall E \cap F=\varnothing\{\tau(E \cup F)+\tau(E \cap F) \geq \tau(E)+$ $\tau(F)\}$;

5. subadditive: $\forall E \cap F=\varnothing\{\tau(E \cup F)+\tau(E \cap F) \leq \tau(E)+$ $\tau(F)\}$

6. symmetric: $|E|=|F| \rightarrow \tau(E)=\tau(F)$;

7. Boolean: $\tau(E)=0 \vee 1$

It represents the human behavior that transcends the 'syntactic' boundaries of variable data with the respect of semantics and pragmatics.

The measure $\tau$ corresponding to above conditions is called cognitive measure - a true-false measure in the measurable space $(\mathrm{X}, \mathbb{A})$, which we call a non-additive measure also. When it is used to define a function such as the Sugeno integral [13], these properties will be crucial in understanding the function's behavior. Let $\lambda \neq 0$ and $\left\{E_{1}, E_{2}, \ldots, E_{n}\right\}$ be a disjoint class of sets in $\mathbb{A}$; we have:

$\tau\left(\bigcup_{i=1}^{n} E_{i}\right)=\frac{1}{\lambda}\left\{\prod_{i=1}^{n}\left[1+\lambda \tau\left(E_{i}\right)\right]-1\right\}$

Definition A set function $\tau$ is called a $\lambda$-fuzzy measure on $\mathbb{A}$ iff it satisfies the $\sigma-\lambda$-rule on $\mathbb{A}$, and there at least one set $E \in \mathbb{A}$ such that $\tau(E)<\infty$. A set function, $\tau$, satisfies the $\lambda$-rule (on $\mathbb{A}$ ) iff there exists:

$\lambda \in(-(1 / \sup \tau), \infty) \cup\{0\}$,

where, $\sup \tau=\sup _{E \in \mathrm{A}} \tau(E)$ such that:

$$
\begin{aligned}
& \forall_{E, F \in \mathrm{A}, E \cup F \in \mathrm{A}, E \cap F=\varnothing\{\tau(E \cup F)} \\
& =\tau(E)+\tau(F)+\lambda \tau(E) \tau(F)\}
\end{aligned}
$$

$\tau$ satisfies the finite $\lambda$-rule (on $\mathrm{A}$ ) iff there exists the abovementioned $\lambda$ such that:

$$
\begin{aligned}
& \forall E_{E_{i} \in \mathrm{A}, i=1,2, \ldots, n, E_{i} \cap E_{j}=\varnothing, E_{i} \cup E_{j} \in \mathrm{A}, i \neq j} \tau\left(\bigcup_{i=1}^{n} E_{i}\right) \\
& \quad=\left\{\begin{array}{l}
\frac{1}{\lambda}\left\{\prod_{i=1}^{n}\left[1+\lambda \tau\left(E_{i}\right)\right]-1\right\} \text { as } \lambda \neq 0 \\
\sum_{i=1}^{n} \tau\left(E_{i}\right) \text { as } \lambda=0
\end{array}\right.
\end{aligned}
$$


$\tau$ satisfies the finite $\sigma-\lambda$-rule (on A) iff there exists the above-mentioned $\lambda$ such that:

$$
\begin{aligned}
& \forall_{E_{i} \in \mathrm{A}, i=1,2, \ldots, n, E_{i} \cap E_{j}=\varnothing, E_{i} \cup E_{j} \in \mathrm{A}, i \neq j} \tau\left(\bigcup_{i=1}^{\infty} E_{i}\right) \\
& \quad= \begin{cases}\frac{1}{\lambda}\left\{\prod_{i=1}^{\infty}\left[1+\lambda \tau\left(E_{i}\right)\right]-1\right\} & \text { as } \lambda \neq 0 \\
\sum_{i=1}^{\infty} \tau\left(E_{i}\right) \quad \text { as } \lambda=0\end{cases}
\end{aligned}
$$

When $\lambda=0$, the $\lambda$-rule, the finite $\lambda$-rule and $\sigma-\lambda$-rule is the additivity, the finite additivity and the $\sigma$-additivity, respectively. Note that if $\mathbb{A}=\Re$ is a non-empty class such that $E, F \in \Re, E \cup F \in \Re$ and $E-F \in \Re$ and $g$ satisfies the $\lambda$-rule, then $\tau$ satisfies the finite $\lambda$-rule.

Sugeno $\lambda$-measure. The Sugeno $\lambda$-measure [13], is a special case of $f u z z y$ measures defined iteratively. It has the following definition: let $\mathrm{X}=\left\{x_{1}, x_{2}, \ldots, x_{n}\right\}$ be a finite set and let $\lambda \in(-1,+\infty)$. A Sugeno $\lambda-$ measure is a function $\tau$ from $2^{X}$, (i.e., $\{0,1\}^{X}$ is the set of all functions from $X$ to $\{0,1\}$ ) to $[0,1]$ with properties:

$$
\begin{aligned}
\tau(X) & =1 \wedge(E, F \subseteq X \wedge E \cap F=\varnothing) \rightarrow \tau(E \cup F) \\
& =\tau(E)+\tau(F)+\lambda \tau(E) \tau(F)
\end{aligned}
$$

where $\lambda$ is determined by:

$1+\lambda=\left\{\prod_{i=1}^{n}\left[1+\lambda \tau\left(E_{i}\right)\right]\right\} ; \quad \lambda_{\in}(-1, \infty) \wedge \lambda \neq 0$

An evaluation undertaken by a single expert is always influenced by his/her subjectivity. However, we can imagine that each quality factor $\mathrm{x}_{i}$ of a given object also has its inherent quality index $\mathrm{h}\left(\mathrm{x}_{i}\right) \in[0,1], i=1,2, \ldots, n$. That is, we assume the existence of an objective evaluation function $h$ : $X \rightarrow[0,1]$. The most ideal evaluation, $E^{*}$, for the quality of the object is the fuzzy integral: $\mathrm{E}^{*}=f h \mathrm{~d} g$ of this function $h$ with respect to the importance measure $g$, which we call the objective synthetic evaluation. Wang (1984-1992) [14] introduced a generalization of the fuzzy integral. It is nonlinear functional, where the integral is defined over measurable sets. Let $A \in \mathrm{A}, \mathrm{A}$ is a $\sigma$-algebra of sets in $\wp(X) ; f \in \mathbf{F}$, $\mathbf{F}$ is the class of all finite non-negative measurable functions defined on measurable space $(\mathrm{X}, \mathbb{A})$. For any given $f \in \mathbf{F}$, we write $F_{\alpha}=\{x \mid f(x) \geq \alpha\}, F_{\alpha+}=\{x \mid f(x)>\alpha\}$, where $\alpha \in[0, \infty]$. Let $F_{\alpha}$ and $F_{\alpha+}$ be called an $\alpha$-cut and strict $\alpha$-cut of $f$, respectively. Instead of the importance measure $g$, the fuzzy integral of $f$ on $A$ with respect to true-false measure, $\tau$, is denoted by $f_{A} f \mathrm{~d} \tau$. To simplify the calculation of the fuzzy integral, for a given $(X, \mathbb{A}, \tau), f \in \mathbf{F}$ and $A \in \mathbb{A}$, we have:

$$
\begin{aligned}
& f_{A} f \mathrm{~d} \tau=\sup _{\alpha \in \mathrm{ALFA}}\left[\alpha \wedge \tau\left(\mathrm{A} \cap \mathrm{F}_{\alpha}\right)\right] \\
& \mathrm{ALFA}=\left\{\alpha \mid \alpha \in[0, \infty], \tau\left(\mathrm{A} \cap \mathrm{F}_{\alpha}\right)>\tau\left(\mathrm{A} \cap \mathrm{F}_{\beta}\right),\right. \\
& \quad \text { for }- \text { any }: \beta>\alpha\}
\end{aligned}
$$

The True-false measure referred as fuzzy measure enables us to represent the semantic and pragmatic aspects of knowledge through the human pragmatic competences. These can be estimated by how much it resonates with our being through an internal processing (feeling) of the human mind. It is the result of quantity expressed by numbers through cognitive measures, quality expressed by words through metaconcepts and the human thought through cognitive information processing together held in relation to Frege's concept of sense and denotation of sentences and Lukasiewicz's multivalued logic.

\section{Determining the relative density of sands, $I_{D}$, using CPT data}

We can use $I_{D}$, for example, to indicate the state of denseness of sand soil. Current knowledge on the subject of sand consolidation is limited and qualitative. For example, soil consolidation states, $\mathrm{s}$, are presented by loose (1-loose/Low consolidation) for $0<\mathrm{I}_{\mathrm{D}} \leq 0.33$, moderately consolidated (m-medium consolidation) for $0.33<\mathrm{I}_{\mathrm{D}} \leq 0.67$, or highly consolidated ( $\mathrm{h}-$ high consolidation) for $\mathrm{I}_{\mathrm{D}}>0.67$, which are shown in the Fig. 6.

The counter-concept, loose or medium or high consolidation, is represented logically in the form:

$s \in\{l \vee m \vee h\}$

where, $\vee$ denotes alternative operator (or) in two-valued logic. Then, we have loose consolidation for $\mathrm{I}_{\mathrm{D}}=0.33$, medium consolidation for both $\mathrm{I}_{\mathrm{D}}=0.34$ and $\mathrm{I}_{\mathrm{D}}=0.67$. It, however, is hard to recognize, for both $\mathrm{I}_{\mathrm{D}}=0.34$ and $\mathrm{I}_{\mathrm{D}}=$ 0.67 , the same conclusions-medium consolidation.

From engineering experiences, for $I_{D}=0.34$, it really is neither loose nor medium consolidation, but a third option determined by cognitive evaluations formulated in the engineer's head that transcends both loose and medium consolidation depending on his/her intuition and experiences. That is to say, engineers have certainly in mind an infinite number of

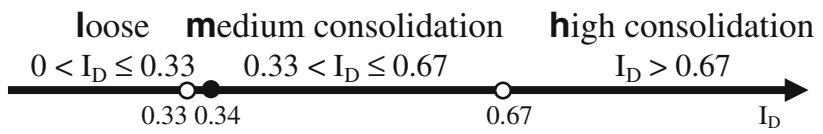

Fig. 6 Presentation of soil consolidation states based on 'true or false' philosophy 
thoughts, which cannot be expressed by counter-concepts, in the framework of syntactic restriction of the common language, using words, loose or medium consolidation, and Boolean numbers. It we can represent rather by the metaconcept, loose-medium, with its denotation identified by an infinite number of true-false values referred as cognitive measures depending on perceptions originated from intuition and experiences of engineers. For example, for $\mathrm{I}_{\mathrm{D}}=0.34$, according to Eq. (3) we have:

$$
\text { loose-medium } \mid \tau(\mathrm{l})=0.8 \wedge \text { loose-medium } \mid \tau(\mathrm{m})=0.2 \text {. }
$$

It is concerned with a new approach to determine the relative density of soil, $\mathrm{I}_{\mathrm{D}}$, based on true-false philosophy that is presented below in the Sect. 6.2.

\subsection{Determining relative density of sands using fuzzy philosophy}

Traditionally, determining of the relative density of sands, $\mathrm{I}_{\mathrm{D}}$, using the Cone Penetration Test, CPT, is based on three compressibility qualifiers as low or medium or high. They are presented by interval numbers established depending on the current knowledge of the friction ratio, $r$, and the compressibility relationship. That are low, $\mathrm{L}$, for $r=[0 \div 0.3] \%$, medium, $\mathrm{M}$, for $r=[0.3 \div 0.7] \%$ and high, $\mathrm{H}$, for $r=[0.7$ $\div 1.0] \%$, in which $r=f_{s} / q_{c}[\%]$, where $f_{s}$ denotes the sleeve friction, $q_{c}$ denotes the cone-tip resistance. It refers as three base correlations, which is graphically presented in the Fig. 7.

According to the fuzzy approach, as in [15], three compressibility qualifiers: low (L), medium (M) and high $(\mathrm{H})$ are represented by three fuzzy sets and expressed by three membership functions $\mu(\mathrm{L}), \mu(\mathrm{M})$ and $\mu(\mathrm{H})$, which are established on the base of engineering judgments. Those three fuzzy sets low, medium and high are shown in the Fig. 8.

To determine $\mathrm{I}_{\mathrm{D}}$, a weighted aggregation technique has been used to combine three base correlations, determined

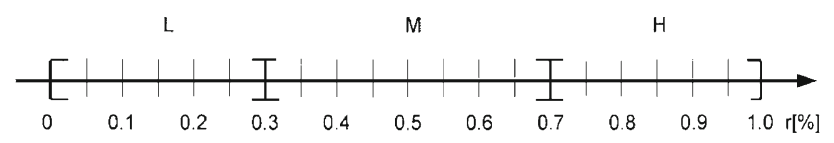

Fig. 7 Sand compressibility in the framework of beingor nonbeing philosophy

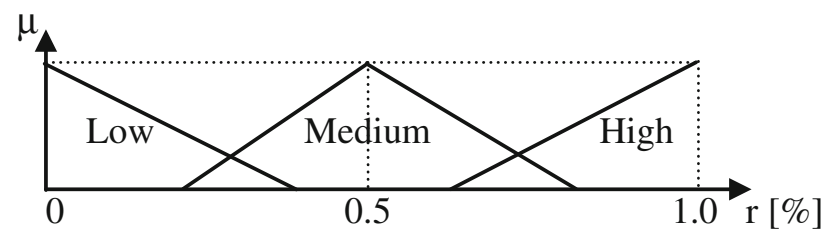

Fig. 8 Three levels of compressibility in the framework of membership-non-membership philosophy from the correlations, which are defined for sands of low, medium and high compressibility, respectively. The relative density $\mathrm{I}_{\mathrm{D}}$ obtained from these base correlations, presented by Juang et. al. [15], is then aggregated as follows:

$\mathrm{I}_{\mathrm{D}}=\mathrm{I}_{\mathrm{D}}^{\mathrm{L}} \mathrm{w}^{\mathrm{L}}+\mathrm{I}_{\mathrm{D}}^{\mathrm{M}} \mathrm{w}^{\mathrm{M}}+\mathrm{I}_{\mathrm{D}}^{\mathrm{H}} \mathrm{w}^{\mathrm{H}}$

where, $\mathrm{I}_{\mathrm{D}}^{k}, k=\mathrm{L}, \mathrm{M}, \mathrm{H}$ are relative densities, determined from the correlations defined for sands of low, medium and high compressibility, respectively (they usually relate the cone-tip resistance $\left(q_{c}\right)$ to $\mathrm{I}_{\mathrm{D}}$ with consideration of effective overburden stress $\left(\sigma_{v}^{\prime}\right)$ and soil compressibility); $w^{k}$, denotes weights which are determined based on a "similarity" measure of three predefined levels of compressibility. The result based on fuzzy logic, obtained by Juang et al. is $\mathrm{I}_{\mathrm{D}}^{J}=41 \%$.

As the above aggregation, Eq. (22), is based on an implicit assumption that these effects of three compressibility levels $(\mathrm{L}, \mathrm{M}, \mathrm{H})$ are viewed as additive. This assumption, however, is not always reasonable as indicated by Wang and Klir [14]. On the other hand, although the general idea of degrees of membership agrees well with the general idea of truefalse/one philosophy, we have to see in this approach that the dividing compressibility of sands into three levels, which are described by three fuzzy sets low, medium and high separately or partly overlapped, does not agree with the "one" philosophy. In this case, saying as Hegel, we are constantly using our one reality to subdivide and rearrange new sets of ones.

\subsection{Determining relative density of sands based on True-False philosophy}

Let a set function, $\tau$, be employed as a true-false measure/cognitive measure for available data, which is derived from the engineer's perceptions in the pragmatic and semantic respects depending on their intuition and experiences. It is a qualitative-quantitative evaluation used for the metaconcept, in which, three levels of compressibility, according to Hegel's philosophy, are undivided and expressed as 'one': Low-Medium-High, (L-M-H). It refers as appearance of the so-called meta-state of sand (three states, low, medium and high compressibility together, which is not loose and neither medium nor High). Three levels of compressibility are presented graphically for any value, $r_{i}$, in the Fig. 9 .

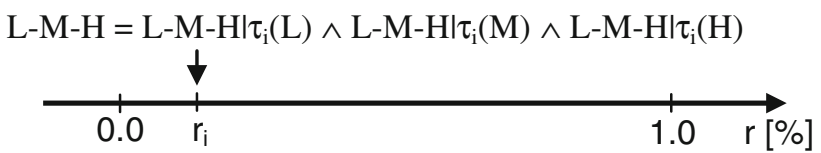

Fig. 9 Three levels of compressibility in the framework of true-false philosophy 
This refers to the appearance of one state $\mathrm{L}-\mathrm{M}-\mathrm{H}$ presented in the form:

$\mathrm{L}-\mathrm{M}-\mathrm{H}=\mathrm{L} \wedge \mathrm{M} \wedge \mathrm{H}$

A set function, $\tau(\mathrm{L}-\mathrm{M}-\mathrm{H})$, is employed as a true-false measure (cognitive measure)—non-additive measure, i.e.,

$\tau(\{\mathrm{L} \cup \mathrm{M} \cup \mathrm{H}\}) \neq \tau(\mathrm{L})+\tau(\mathrm{M})+\tau(\mathrm{H})$.

We can distinguish different states depending on variable true-false measures, which is non-additive measure defined by:

$\tau: R \rightarrow[0,1]$

It is a qualitative-quantitative evaluation of $R, R=$ $\left\{r_{i}\right\}, i=1,2, \ldots, n$, for compressibility of sands in terms of multiple quality factors expressed by true-false measures, $\tau$, As a result, the compressibility level of sands is lowmedium-high, in which we can recognize low from medium, medium from high and vice versa through variable true-false measures $\tau(k), k=\mathrm{L}, \mathrm{M}, \mathrm{H}$ and true-false analysis, as in [16]. A true-false value of each predefined level of compressibility (low, medium, high) in respect of the given or actual compressibility, $r_{a}$, is determined on the base of "difference" of $r_{a}$ and the numbers represented the low, medium, high levels of compressibility, respectively. For most NC sands, according to Robertson and Campanella (1985), the predefined value of $r$ for medium compressibility $r^{*}(\mathrm{M})$ is about $0.5 \%$, but for sands of low compressibility $r^{*}(\mathrm{~L}) \approx 0 \%$ and for sands of high compressibility it is $r^{*}(\mathrm{H}) \approx 1 \%$. Hence, the difference of $k$-level compressibility and number representing the given or actual compressibility, $r_{a},\left(\operatorname{diff}_{r a}(k)\right)$ is defined as follows:

$$
\begin{aligned}
\operatorname{diff}_{r a}(k)= & \mid r_{a}-r^{*}(k) ; \tau\left(r^{*}(k)\right)=\text { maximum } \mid ; \\
& r^{*}(k) \in k \subset R
\end{aligned}
$$

This is a distance between number, $r_{a}$, representing the actual compressibility and number $r^{*}(k)$, which represents the most probable value that reaches a maximum value for sand of low, medium and high compressibility. This distance is used as a means of measuring how close the actual compressibility is to each of the predefined levels of compressibility. For subjective evaluation: $\tau\left(r_{1}\right)=\tau\left(r_{2}\right)=\cdots=\tau\left(r_{n}\right),\left(r_{x} \in\right.$ $\left.k_{j}, x=1,2, \ldots, n\right)$ we have:

$\operatorname{diff}_{r a}(k)=0$

Smaller distance indicated a higher degree of similarity, and the level of compressibility corresponding to a higher is assigned a greater value of truth, which is represented as true-false function, $\tau$ : $\tau(k)=1-\operatorname{diff}_{r a}(k)$

From that we can construct the $\lambda$-true-false measure for all the other subsets of set $X, X=\{\mathrm{L} \cup \mathrm{M} \cup \mathrm{H}\}$ using Sugeno $\lambda$ measure. Then, we can calculate the $\lambda$-true-false measures, $\{\tau(\mathrm{L} \cup \mathrm{M}), \tau(\mathrm{L} \cup \mathrm{H})$ and $\tau(\mathrm{M} \cup \mathrm{H})\}$ by

$$
\begin{aligned}
\tau(R) & =\tau(\{\mathrm{L} \cup \mathrm{M} \cup \mathrm{H}\})=1 \\
& =\frac{1}{\lambda}\left\{\prod_{i=1}^{n}\left[1+\lambda \tau\left(\left\{k_{j}\right\}\right)\right]-1\right\}
\end{aligned}
$$

From that, value $\lambda$ can be found by:

$$
\begin{aligned}
& \lambda=\left\{\prod_{i=1}^{n}\left[1+\lambda \tau\left(\left\{k_{j}\right\}\right)\right]\right\}-1 \\
& \tau\left(k_{i} \cup k_{j}\right)=\tau\left(k_{i}\right)+\tau\left(k_{j}\right)+\lambda \tau\left(k_{i}\right) \tau\left(k_{j}\right) \\
& \quad \text { for } k_{i}, k_{j} \in R, k_{i} \cap k_{j}=\varnothing
\end{aligned}
$$

i.e., sand, having the compressibility level $k$ is intuitively assigned to this true-false measure (cognitive measure) $\tau(k)$. From that, we can construct the $\lambda$-true-false measure for all the other subsets of set $X, X=\{\mathrm{L} \cup \mathrm{M} \cup \mathrm{H}\}$. Then, we can calculate the $\lambda$-true-false measures, $\{\tau(\mathrm{L} \cup \mathrm{M}), \tau(\mathrm{L} \cup$ $\mathrm{H})$ and $\tau(\mathrm{M} \cup \mathrm{H})\}$ by Eqs. $(28,29,30)$ for different subsets $\{(L \cup M),(L \cup H)$ and $(M \cup H)\}$. In addition, we support here the given evaluations by three experts expressed by: $\tau_{i}^{*}(k), i=1,2,3$. The relative densities, $\mathrm{I}_{\mathrm{D}}(k)$ are determined from the correlation defined for sands of low, medium and high compressibility, respectively. Scores of these may be regarded as a measurable function $f(k)$ defined on $(R, \wp(R))$ such that $f(k) \in[0,1]$ for each $k \in R$. Then, relative density, $\mathrm{I}_{\mathrm{D}}$, by a cognitive processing with both physical and mental data using Sugeno type integral, as in [13], of a measurable function $f(k), f(k) \in F_{\alpha}$ with respect to the true-false measure, $\tau$, in the form:

$\mathrm{I}_{\mathrm{D}}=\int f(k) \mathrm{d} \tau=\sup _{\alpha \in[0, \infty]}\left[\alpha \wedge \tau\left(R \cap F_{\alpha}\right)\right]$,

where,

$F_{\alpha}=\{k \mid f(k) \geq \alpha\}, \quad k \subset R ;$

in which, $R=\{\mathrm{L}, \mathrm{M}, \mathrm{H}\} ; \mathrm{L}, \mathrm{M}, \mathrm{H}$ represent the loose, medium and high states of sands. The evaluations of three experts are shown in the following table.

\section{Data and results:}

1. Data from the Texas A\&M University National Geotechnical Experimentation Site (Gibbens and Briaud 1994), four CPT sounding are obtained at this site. The data from four CPT sounding along with other pertinent data used for determining relative density are listed in Table 1. 
Table 1 CPT data from Texas A\&M University site (Gibbens and Briaud 1994)

\begin{tabular}{llllll}
\hline CPT number & Depth $(\mathrm{m})$ & $\sigma_{v}^{\prime}(\mathrm{kPa})$ & $q_{c}(\mathrm{kPa})$ & $f_{s}(\mathrm{kPa})$ & $r_{a}(\%)$ \\
\hline 1 & 3.0 & 46.5 & 6,260 & 38 & 0.61 \\
2 & 3.0 & 46.5 & 6,590 & 35 & 0.53 \\
5 & 3.0 & 46.5 & 6,590 & 43 & 0.65 \\
6 & 3.0 & 46.5 & 6,190 & 37 & 0.60 \\
\hline
\end{tabular}

Numerical data: $\sigma^{\prime}{ }_{\nu}=46.5(\mathrm{kPa}), q_{c}=6260(\mathrm{kPa}), f_{s}=$ $38(\mathrm{kPa})$ and the given friction ratio, $r_{a}=0.61 \%$, are used for determining relative density, $\mathrm{I}_{\mathrm{D}}$ of sand and to illustrate the new approach.

According to Robertson and Campanella [15], the value, $r$, increases with increasing sand compressibility. For most NC sands, the $r_{m}^{*}$ value for medium compressibility is about $0.5 \%$, but the $r_{L}^{*}$ value is almost 0 for sands of low compressibility and $r_{H}^{*}=1 \%$ (or more) for sands of high compressibility. For purpose of verifying the proposed model, the CPT data at the depth of $3 \mathrm{~m}$ are considered to be appropriate. Difference of low level compressibility $(L)$ and the number represented the actual compressibility, $r_{a}, r_{a}=$ $0.61 \%$, for example, is determined as follows:

$\operatorname{diff}(L)_{0.61}=0.61-0.0=0.61$

Then, from Eq. 27 we have:

$\tau(L)=1-0.61=0.39$

Repeating this process for all other levels of compressibility, we obtain $\tau(M)=0.89$, and $\tau(H)=0.61$. From that we can construct the $\tau \lambda$-fuzzymodal measure for all other subsets of set $R, R=\{L, M, H\}$ as follows: firstly, $\lambda$ parameter is determined using Eq. 29 as:

$\lambda+1=(1+0.39 \lambda)(1+0.89 \lambda)(1+0.61 \lambda)$

The solution of this equation according to the unique root greater than -1 is -0.99 . And the $\tau_{\lambda}$-fuzzy measures for other subsets of set $R$ are defined using Eq. 28, for example,

$\tau(\mathrm{L} \cup \mathrm{M})=0.39+0.89-0.99(0.39) 0.89=0.93$

By similar ways, we can obtain $\tau(L \cup H)=0.76$; $\tau(M \cup$ $H)=0.96$. Finally, value $\mathrm{I}_{\mathrm{D}}$ is calculated by Eqs. (31), (32).
For example, for value $r_{a}=0.61$ and various values of relative densities for separately states: loose, medium and high: $\mathrm{I}_{\mathrm{D}}^{\mathrm{L}}=0.526, \mathrm{I}_{\mathrm{D}}^{\mathrm{M}}=0.549, \mathrm{I}_{\mathrm{D}}^{\mathrm{H}}=0.575$, determined from the correlations defined for sand of low, medium, and high compressibility, respectively, we have:

$$
\begin{aligned}
\mathrm{I}_{\mathrm{D}}=f f(k) \mathrm{d} \tau= & {[0.526 \wedge \tau(\mathrm{L} \cup \mathrm{M} \cup \mathrm{H})] } \\
& \vee[0.549 \wedge \tau(\mathrm{M} \cup \mathrm{H})] \\
& \vee[0.575 \wedge \tau(H)] \\
= & (0.526 \wedge 1) \vee(0.549 \wedge 0.96) \\
& \vee(0.575 \wedge 0.61)=0.575 .
\end{aligned}
$$

where ' $\wedge$ ' and ' $\vee$ ' denote 'min' and 'max' operations, respectively. The above process is repeated for data from other CPTs and the complete results are shown in Table 2.

In which, $\mathrm{I}_{\mathrm{D}}$ values is determined by the proposed model using cognitive measures and fuzzy/true-false integral, I'D values are the results obtained from Juang et al. (1996). Thus, $I_{D}$ values determined by the proposed model in all four CPTs examined here agree well with the results obtained by Juang et al. [15].

2. Data from two sites, the Hunter's point, California site with their scores, for example, are given for available CPT data: $\sigma_{v}^{\prime}=81(\mathrm{kPa}), q_{c}=5,030(\mathrm{kPa}), f_{s}=3(\mathrm{kPa})$ and the given friction ratio, $r_{a}=0.06 \%$ are used for determining relative density, $\mathrm{I}_{\mathrm{D}}$ of sand.

Details on the Hunter's point, California site are described in a Federal Highway Administration (FHwA) report by Dimillio et al. (1987) represented in Juang et al. [15], which documents the results of a pile prediction event. Although a thorough site investigation including several field tests was conducted, only those data required in the present study are summarized here. By the same way presented for Texas site, the predicted I* values obtained from the proposed model using $\lambda$-true-false measures and fuzzy/true-false integral. To study the influence of subjective biases of the individual experts and to get a more reasonable evaluation, we can use an arithmetic average of scores given by a number of experts. We support, for this case, the given evaluations by three experts expressed by: $\tau_{\mathrm{i}}^{*}(k), i=1,2,3$. From that we can construct the $\lambda$-true-false measure for all the other subsets of set

Table 2 Determination of relative density, $\mathrm{I}_{\mathrm{D}}$, by $\tau_{\lambda}$-fuzzy measure and fuzzy integral and $\mathrm{I}_{\mathrm{D}}^{\prime}$ by Juang's et al. method (Texas A\&M university site)

\begin{tabular}{lllllllll}
\hline CPT nr. & $\tau(\mathrm{L})(-)$ & $\tau(\mathrm{M})(-)$ & $\tau(\mathrm{H})(-)$ & $\tau_{(\mathrm{L} \cup \mathrm{M})}(-)$ & $\tau_{(\mathrm{L} \cup \mathrm{H})}(-)$ & $\tau_{(\mathrm{M} \cup \mathrm{H})}(-)$ & $r_{\mathrm{a}}(\%)$ & $\mathrm{I}_{\mathrm{D}} / I_{D}^{\prime}(\%)$ \\
\hline 1 & 0.39 & 0.89 & 0.61 & 0.93 & 0.76 & 0.96 & 0.61 & $57.5 / 55$ \\
2 & 0.57 & 0.97 & 0.53 & 0.99 & 0.80 & 0.98 & 0.53 \\
5 & 0.35 & 0.85 & 0.65 & 0.92 & 0.78 & 0.97 & 0.65 \\
6 & 0.40 & 0.90 & 0.60 & 0.96 & 0.77 & 0.98 & 0.60 \\
\hline
\end{tabular}


Table 3 Qualitative-quantitative evaluations and numerical results, $\mathrm{I}_{\mathrm{D}}^{*}$

\begin{tabular}{llllllll}
\hline Expert & $\tau_{\mathrm{i}}^{*}$ & $\tau_{\mathrm{i}}^{*}$ & $\tau_{\mathrm{i}}^{*}$ & $\tau(\mathrm{L} \cup \mathrm{M})$ & $\tau(\mathrm{L} \cup \mathrm{H})$ & $\tau(\mathrm{M} \cup \mathrm{H})$ & $\mathrm{I}_{\mathrm{D}}^{*}(\%)$ \\
\hline 1 & 0.80 & 0.30 & 0.10 & 0.95 & 0.85 & 0.38 & 41.0 \\
2 & 0.80 & 0.50 & 0.10 & 0.98 & 0.84 & 0.56 & 42.8 \\
3 & 0.80 & 0.30 & 0.20 & 0.93 & 0.88 & 0.46 & 42.8
\end{tabular}

$X, X=\{\mathrm{L} \cup \mathrm{M} \cup \mathrm{H}\}$. Then, we can calculate the $\lambda$-true-false measures for different subsets $\{(\mathrm{L} \cup \mathrm{M}),(\mathrm{L} \cup \mathrm{H})$ and $(\mathrm{M} \cup \mathrm{H})\}$ and the true-false measures of subsets $\{\tau(\mathrm{L} \cup \mathrm{M}), \tau(\mathrm{L} \cup \mathrm{H})$ and $\tau(\mathrm{M} \cup \mathrm{H})\}$ by Eqs. $(28,29,30)$, and $I_{\mathrm{D}}^{*}$ by Eqs. (31, 32). Three given expert's evaluations and obtained results are shown in the following Table 3.

Obviously, the changes of results $\mathrm{I}_{\mathrm{D}}^{*}$, depending on changes of $\left\{\tau^{*}(\mathrm{~L}), \tau^{*}(\mathrm{M}), \tau^{*}(\mathrm{H}), \quad \tau(\mathrm{L} \cup \mathrm{M}), \quad \tau(\mathrm{L} \cup \mathrm{H})\right.$, $\tau(\mathrm{M} \cup \mathrm{H})\}$, confirm the requirement that the relative true-false measures, $\tau$, of the compressibility are taken into account in the true-false-integral operator. To reduce the influence of qualitative biases of independent experts and to obtain a more reasonable evaluation of $\mathrm{I}_{\mathrm{D}}^{*}$, we can use an arithmetic average of the results obtained from the three experts. That is:

$(0.41+0.428+0.428) / 3=42.2 \%$.

Although those $\mathrm{I}_{\mathrm{D}}^{*}$ values based on "one" philosophy agree well with the results based on fuzzy logic, as in [15], $\mathrm{I}_{\mathrm{D}}^{J}=$ $41 \%$. These results of both methods are depended on the expert's qualitative-quantitative evaluations also, which we can improve by a process of inductive thinking.

\section{Conclusions}

To recognize the real world, we are confined by syntactic limits in the framework of binary philosophy. It is only a matter of habit, which we should change in the future from dualism with counter-concepts to a monism with new concepts named meta-concepts. It is really that, to describe the real systems like soil systems, a formulation of the counter-concept loose or consolidation based on available data resulted from physical experiments is insufficient. We need meta-concepts that transcend limitations of both common words and Boolean numbers. The meta-concept: lowmedium-high, derived from perceptions of engineers rather than logical reasoning, represents a third option that transcends both low, medium and high.

These expressive needs require a theory of cognitive measures, a new field of mathematics for denotation computing and their integration with the achievements of modern information and communication technologies to create the socalled cognitive processing of information for various fields of science. These areas of research that require more intel- ligence and contemplation for "the truth that comes from the future" should require being-nonbeing philosophy and multi-valued logic.

Generally we represent, in this paper, a new approach for cognitive information processing to expand the syntactic boundaries under the respects of semantics and pragmatics of the available data. It is applied to recognize the unknown reality of the real world rather than application of an approximation approach to improve the known reality through external information processing. It is based rather on the integration of modern philosophy, epistemology, logic, denotational mathematics and cognitive informatics using engineer's perceptions rooted from intuition, commonsense and experiences without logical reasoning based on binary logic.

Acknowledgments I would like to express many thanks for the support of the National Center of Sciences in Krakow and WM University of Olsztyn, Poland, grant nr. NN 506215440.

Open Access This article is distributed under the terms of the Creative Commons Attribution Noncommercial License which permits any noncommercial use, distribution, and reproduction in any medium, provided the original author(s) and source are credited.

\section{References}

1. Frege, F.L.G.: Über Sinn und Bedeutung. In: Zeitschrift für Philosophie und philosophische Kritik, vol. 100, pp. 25-50 (Translated as 'On Sense and Reference' by M. Black in Translations from the Philosophical Writings of Frege, G., Geach, P., Black, M. (eds. and trans.) 3rd edn. Blackwell, Oxford (1980))

2. Stanford Encyclopedia of Philosophy. http://plato.stanford.edu/ entries/frege

3. Thuc, N.D.: Lich su triet hoc dong Phuong (History of Eastern philosophy). Nha xuat ban van hoa, Hanoi, Viet Nam (1963)

4. Giang, T., Can. N.D.: Trang Tu (莊 周- Zhuang Zhou)-Nam Hoa Kinh t.1, 2; nha xuat ban Hanoi, Viet Nam (1992)

5. Suzuki, D.T.: C'est ici le paradoxe philosophique le plus profond; L'Essence du Bouddhisme, p. 88, Paris (1962)

6. Hunefeldt, T., Brunetti, R.: Artificial intelligence as "theoretical psychology" : Christopher Longguet-Higgins' contribution to cognitive science. Cognitive Processing International Quarterly of Cognitive Science, Marta Olivetti Belardinelli and Springer (2004)

7. Łukasiewicz, J.: O logice trójwartościowej (about three-valued logic). Ruch Filozoficzny (Philosophical movement), No. 5, pp. 169-170 (1920) (in Poland)

8. Zadeh, L.A.: Fuzzy sets. Inf. Contr. 8(3), 338-353 (1965)

9. Heyting, A.: Intuitionism, An Introduction (Amsterdam, 1965) (1965)

10. Heyting, A. (ed.): L E J Brouwer, Collected Works 1. Philosophy and Foundations of Mathematics (Amsterdam, 1975) (1975)

11. Perrin, L.: Henri Lebesgue: Renewer of Modern Analysis. In: Le Lionnais, François. Great Currents of Mathematical Thought, vol. 1, 2nd edn. Courier Dover Publications. Plato J.V.: Creating Modern Probability, Its Mathematics. Physics and Philosophy in Historical Perspective. Cambridge Uni. Press 1994 (2004)

12. Kharazishvili, A.B.: On almost measurable real-valued functions. Studia Scientiarum Mathematicarum Hungarica 47(2), 257-266 (2010) NULL 
13. Sugeno, M.: Fuzzy Measures and Fuzzy Integrals: A Survey, pp. 89-102. North-Holland, Fuzzy Automata and Decision Processes. Amsterdam (1977)

14. Wang, Z., Klir, G.J.: Fuzzy Measure Theory. Plenum Press, N.Y. (1992)

15. Juang, C.H., Huang, X.H., Holtz, R.D., Chen, J.W.: Determining relative density of sands from CPT using fuzzy sets. J. Geol. Eng. 122, 1-5 (1996)
16. Tran, C.: Dealing With Geotechnical Uncertainties by Being-NonBeing Philosophy and Multi-Valued Logic. (book) pub. WM Univ, Olsztyn, Poland (2012) 